\title{
Correction to: Informality and Disaster Vulnerability: A Preliminary Exploration
}

\author{
Lopamudra Banerjee and Snehashish Bhattacharya
}

\section{Correction to:}

Chapter "Informality and Disaster Vulnerability:
A Preliminary Exploration" in:

S. Bandyopadhyay and M. Dutta (eds.),

Opportunities and Challenges in Development, https://doi.org/10.1007/978-981-13-9981-7_22

The original version of the book was inadvertently published with an incorrect abstract in the Chapter "Informality and Disaster Vulnerability: A Preliminary Exploration". The book has now been updated with the changes. 